\title{
The effects of nuclear reprogramming on mitochondrial DNA replication.
}

Richard D.W. Kelly ${ }^{1,2}$, Huseyin Sumer ${ }^{1}$, Matthew McKenzie ${ }^{1}$, Joao Facucho-Oliveira ${ }^{2,3}$, Ian A. Trounce ${ }^{4}$, Paul J. Verma ${ }^{1}$, Justin C. St. John ${ }^{1,2}$

${ }^{1}$ Centre for Reproduction and Development, Monash Institute of Medical Research, Monash University, 27-31 Wright Street, Clayton, Victoria 3168, Australia.

${ }^{2}$ Mitochondrial and Reproductive Genetics Group, Clinical Sciences Research Institute, Warwick Medical School, The University of Warwick, Clifford Bridge Road, Coventry, CV2 2DX, UK.

${ }^{3}$ Current address: Centre for Molecular and Structural Biomedicine, FCT Bld. 8, Campus of Gambelas, University of Algarve, Faro, Portugal.

${ }^{4}$ Centre for Eye Research Australia, Department of Ophthalmology, University of Melbourne, Royal Victorian Eye and Ear Hospital, East Melbourne, Vic., Australia.

Correspondence: Justin St. John, Centre for Reproduction and Development, Monash Institute of Medical Research, Monash University, 27-31 Wright Street, Clayton, Victoria 3168, Australia. Email: justin.stjohn@monash.edu

\section{Running title: Reprogramming and mtDNA}

\section{Disclosures:}

The authors indicate no potential conflicts of interest. 


\section{Abstract}

Undifferentiated mouse embryonic stem cells (ESCs) possess low numbers of mitochondrial DNA (mtDNA), which encodes key subunits associated with the generation of ATP through oxidative phosphorylation (OXPHOS). As ESCs differentiate, mtDNA copy number is regulated by the nuclearencoded mtDNA replication factors, which initiate a major replication event on Day 6 of differentiation. Here, we examined mtDNA replication events in somatic cells reprogrammed to pluripotency, namely somatic cell-ES (SC-ES), somatic cell nuclear transfer ES (NT-ES) and induced pluripotent stem (iPS) cells, all at low-passage. MtDNA copy number in undifferentiated iPS cells was similar to ESCs whilst SC-ES and NT-ES cells had significantly increased levels, which correlated positively and negatively with Nanog and Sox2 expression, respectively. During pluripotency and differentiation, the expression of the mtDNA-specific replication factors, PolgA and Peo1, were differentially expressed in iPS and SC-ES cells when compared to ESCs. Throughout differentiation, reprogrammed somatic cells were unable to accumulate mtDNA copy number, characteristic of ESCs, especially on Day 6. In addition, iPS and SC-ES cells were also unable to regulate ATP content in a manner similar to differentiating ESCs prior to Day 14. The treatment of reprogrammed somatic cells with an inhibitor of de novo DNA methylation, 5-Azacytidine, prior to differentiation enabled iPS cells, but not SC-ES and NT-ES cells, to accumulate mtDNA copies per cell in a manner similar to ESCs. These data demonstrate that the reprogramming process disrupts the regulation of mtDNA replication during pluripotency but this can be re-established through the use of epigenetic modifiers. 


\section{Introduction}

The maternally inherited mitochondrial genome (mtDNA) encodes 13 of the $90+$ subunits of the electron transfer chain (ETC) [1]. Within the ETC, the process of oxidative phosphorylation (OXPHOS) takes place, which generates the majority of cellular ATP [2]. Functional maturity of OXPHOS complexes I-V (CI-IV) relies upon the expression of nuclear encoded assembly factors coordinating the assembly of OXPHOS subunits encoded by both the nuclear and mitochondrial genomes $[3,4,5]$. Mutations, deletions and depletion of mtDNA, as well as nuclear mutations, can render OXPHOS-dependent cells dysfunctional, which in turn, result in either severely debilitating diseases or lethality $[3,4,5,6,7]$. Transcription and replication of the mitochondrial genome are dependent upon nuclear-encoded transcription and replication factors, which translocate to the mtDNA genome [8]. The key mtDNA replication factors include: the mtDNA specific polymerase, Polymerase Gamma, which consists of a catalytic subunit (POLGA) and its accessory subunit POLGB; mitochondrial transcription factor A (TFAM), which initiates the process of transcription and generates the DNA/RNA hybrid primer that is used to prime mtDNA replication by POLG; the mitochondrial specific helicase, Twinkle; and the mitochondrial single-stranded DNA binding protein (mtSSBP1). Twinkle and mtSSB combine with POLGA and POLGB to generate the minimal mtDNA replisome [9]. Mitochondrial biogenesis, mtDNA gene expression and assembly of the OXPHOS complexes demand coordinated nucleo-mitochondrial communication in order to satisfy cellular demands for ATP $[4,10]$.

MtDNA replication is not initiated during preimplantation development until the final stage, the blastocyst stage, and appears to be restricted to the outer, trophectodermal cells $[11,12]$. The inner cell mass (ICM) cells, which are pluripotent and have the potential to differentiate into all somatic cells and germ cells, exhibit little or no mtDNA replication [11], and possess a mean mtDNA copy number of $4.83 \times 10^{3}$ per cell [13]. It is likely that the ICM cells continue to restrict mtDNA replication, thus mediating the continual dilution of mtDNA in each newly divided cell, as undifferentiated embryonic stem cells (ESC) possess between 30 and 45 copies of mtDNA/cell [14]. During the early stages of ESC differentiation, mtDNA replication remains at very low levels thus establishing the 'mtDNA set point', which provides all specialised cells with the capacity to acquire the appropriate number of mtDNA copies to meet their specific needs for OXPHOS-derived ATP. For example, fully 
differentiated cells, such as skeletal and cardiac muscle cells possess $3650 \pm 620$ and $6790 \pm 920$ mtDNA copies/cell, respectively [15] whilst sheep fetal fibroblasts [16] contain $4241 \pm 411$ copies per cell.

Nevertheless, there is one key mtDNA replication event that takes place on Day 6 of ESC differentiation resulting in a significant increase to $\sim 2370 \mathrm{mtDNA}$ copies/cell [14]. This is then followed by a return to steady state levels of $\sim 13$ copies per cell on Day 7. Day 6 of murine ESC differentiation correlates to mouse embryonic day (E) 7.5, an early stage of organogenesis [17], which appears to be a critical checkpoint for mtDNA content, as homozygous PolgA $A^{-/}$knockout mice do not survive passed this stage of development [18]. On the other hand, their heterozygous counterparts exhibit typical mtDNA depletion-like syndromes, such as cardiomyopathy. Furthermore, the regulation of pluripotency and the expression of PolgA appear to be finely balanced as demonstrated by partial knockdown of PolgA, which results in the loss of pluripotency [14]. This suggests that inconsistencies in this balance and the maintenance of the 'mtDNA set point' will affect a cell's potential to regulate its mtDNA copy number and pluripotent status effectively.

It remains to be determined whether somatic cells that have been reprogrammed to pluripotency are able to establish the 'mtDNA set point' and regulate mtDNA copy number during differentiation [19,20,21]. Reprogramming can be mediated by: i) fusion to ESCs [22,23]; ii) induction with defined factors associated with pluripotency [24,25]; and iii) somatic cell nuclear transfer (NT) [26]. NT embryos display reduced developmental competence due to the incomplete reprogramming of the somatic genome $[26,27,28,29]$. For example, NT embryos continue to express the mtDNA replication factors during preimplantation development, unlike their in vitro fertilised counterparts $[16,30]$ and can preferentially replicate the somatic mitochondrial genome at the expense of the recipient oocyte's mtDNA [16]. However, ESCs derived from NT embryos appear to be more transcriptionally and epigenetically similar to ESCs than iPS cells [31,32]. Additional reprogramming anomalies are observed in induced pluripotent stem (iPS) cells [33,34], which require continuous passaging $[35,36,37]$, serial reprogramming [32] or chromatin-modifying agents $[32,38,39]$ to acquire ESC equivalence. 
In order to determine whether reprogramming can effectively regulate mtDNA copy number during pluripotency and replicate mtDNA at key stages of differentiation, we have investigated these events in low passage somatic cell-embryonic stem (SC-ES) cell hybrids, iPS and NT-ES cells. We have demonstrated that, during pluripotency and the early stages of differentiation, SC-ES cell hybrids and NT-ES cells do not mirror the strict regulation of mtDNA replication exerted on ESCs, although undifferentiated iPS cells do more closely resemble patterns associated with ESCs. Nevertheless, iPS cells do not regulate this effectively during differentiation. Through treatment with 5-Azacytidine, we were able to modulate mtDNA copy number in iPS cells to mimic the early mtDNA replication events of ESCs but not in SC-ES and NT-ES cells. 


\section{Materials and Methods}

\section{Cell culture}

Murine D3 ESCs; iPS 1, 2 and 3 [40], SC-ES 1, 2, 3 and 4 [41], ES-ES [42] and NT-ES [43] cell lines were cultured on gelatin coated flasks $(0.1 \% \mathrm{w} / \mathrm{v})$ in ESC media supplemented with $1000 \mathrm{U} / \mathrm{mL}$ (10 $\mathrm{ng} / \mathrm{ml}$ ) leukemia inhibitory factor (LIF; Chemicon, Millipore UK), as previously described [14]. Additional details can be found within the Supplemental Experimental Procedures.

\section{Spontaneous differentiation}

Undifferentiated ESCs were induced to differentiate using the hanging drop method [44]. Briefly, ESCs were cultured in ESC media minus LIF as $20 \mu$ droplets (approximately 450 cells per drop) on the lid of an inverted Petri dish (Sterilin, Staffordshire, UK) for $48 \mathrm{hr}$ (Days 1 to 2 ) to promote the formation of embryoid bodies. Embryoid bodies were then placed into suspension for a further 5 days at $37{ }^{\circ} \mathrm{C} / 5 \% \mathrm{CO} 2$. They were then plated onto gelatin $(0.1 \%$; Sigma)-coated six-well plates (Nunc, Roskilde, Denmark) and cultured up to Day 14 of differentiation.

Treatment with 5-Azacytidine (5-Aza) and Rhodamine 6G (R6G)

Pluripotent stem cells were cultured in ESC media for $24 \mathrm{hr}$ and then cultured in ESC media supplemented with $10 \mu \mathrm{M}$ 5-Aza for $72 \mathrm{hr}$. Differentiation was induced as described above. For R6G treatment, pluripotent stem cells were cultured in ESC media for $48 \mathrm{hr}$ and then cultured in ESC media supplemented with $1 \mu \mathrm{g} / \mu \mathrm{l}$ R6G for $72 \mathrm{hr}$. For both treatments, the media was changed every 24 hrs.

Immunocytochemistry (ICC)

ICC for 5-Bromo-2'-deoxy-uridine (BrdU; Roche Applied Sciences, Sussex, UK) and MitoTracker Red (Molecular Probes, UK) was performed as previously described [14]. Samples were mounted on slides using mounting medium containing DAPI (Vectashield; Vector Labs, UK). Images were captured using a Carl Zeiss LSM 510 confocal microscope and images analysis was performed using Adobe Photoshop and Zeiss LSM Image Browser (http://www.zeiss.de). 
ATP assay

The ATP content of pluripotent and differentiating cells was determined using the ATP-lite bioluminescence luciferase-based assay (Perlin Elmer, Australia), as described by the manufacturer. Luminescence was measured using a FLUOstar Optima plate reader (BMG Labtech, Germany). Samples were examined in triplicate simultaneously and each time point was measured a minimum of three times. The results were expressed as relative luminescence units compared to undifferentiated ESCs.

Blue-Native Polyacrylamide gel electrophoresis (BN-PAGE)

Whole cell extracts $(100 \mu \mathrm{g})$ were solubilised in $1 \%$ Triton $\mathrm{X}-100$ and separated on a $4-13 \%$ acrylamide gradient gel with a $4 \%$ acrylamide stacking gel, as previously described [45]. After BNPAGE, proteins were transferred onto a polyvinylidene fluoride (PVDF) membrane and probed with primary antibodies against: polyclonal rabbit anti-NADH dehydrogenase (ubiquitin) 1 alpha subcomplex subunit 9 (Complex I) [10]; monoclonal mouse anti-succinate dehydrogenase flavoprotein subunit (Complex II; Molecular Probes, Invitrogen, Australia); monoclonal mouse anti-cytochrome b-c1 complex subunit 1 (Complex III; Molecular Probes, Invitrogen, Australia); and monoclonal mouse anticytochrome c oxidase subunit 1 (Complex IV; Molecular Probes, Invitrogen, Australia) overnight at $4^{\circ} \mathrm{C}$. Proteins bands were detected using anti- goat or rabbit HRP-conjugated secondary antibodies (Sigma, Australia).

\section{RT-PCR and Real Time PCR}

RNA extraction was performed, as previously described in [14], and then reverse transcribed with the BioScript system (Bioline, London, UK). Total DNA was extracted using the Gentra Puregene DNA extraction kit (Qiagen, West Sussex, UK), according to the manufacturer's instructions. The samples were then treated with RNase solution (Qiagen) for $5 \mathrm{~min}$ at $37^{\circ} \mathrm{C}$ to obtain RNA-free DNA. Reactions were performed in a Rotorgene-3000 real-time PCR machine (Corbett Research, Cambridge, UK), as previously described in [14]. Additional details can be found in the Supplemental Experimental Procedures. 
Statistical analysis

All real-time PCR data were normalised to $\beta$-Actin. A one-way ANOVA, in combination with Bonferroni post-hoc tests, was used to determine the differences in mtDNA copy number and expression of the mitochondrial specific replication factors (Tfam, Peo1, mtSsbp1, PolgA and PolgB) between undifferentiated cell types and between days of differentiation within a particular cell type. A two-way ANOVA in combination with Bonferroni post-hoc tests was used to determine differences in Tfam, Twinkle, mtSsbp1, PolgA and PolgB expression and mtDNA copy number between cell types during differentiation. The expression of Oct4, Nanog and Sox2 were ranked by statistical significance $(1=$ increased expression; 0 = non-significant; -1 = reduced expression) and the correlation with mtDNA copy was determined using Pearson's correlation coefficient. Statistical analysis was performed using GraphPad Prism 5.01 (Graph PadSoftware, Inc., San Deigo, CA, http://www.graphpad.com). Data are expressed as mean \pm s.e.m. 


\section{Results}

mtDNA copy number in pluripotent reprogrammed somatic cells

In order to determine whether mtDNA copy number in somatic cells is reset to match that of ESCs following reprogramming, we determined the number of mtDNA copies present in ESCs and mouse embryonic fibroblasts (MEFs), which provided the parental partners for the generation of four somaticES cell hybrid lines (SC-ES 1 to 4). The ESCs had low levels of mtDNA copy number per cell, namely $36.56 \pm 1.79$ whilst the MEFs contained $807.23 \pm 21.8$ copies $(P<0.001$; Fig. $1 \mathrm{~A})$. We then interrogated each of the hybrid lines, which have previously been demonstrated to be pluripotent and capable of differentiating into cell types from all three germ layers [41]. Although each of the lines was generated under the same conditions, we observed that SC-ES 2 and $3(164.46 \pm 4.76$ and $164.41 \pm$ 1.44, respectively) and SC-ES 1 and 4 (251 \pm 3.06 and $284.96 \pm 14.27$, respectively) had very similar levels (Fig. 1A). These two sets were significantly different $(P<0.001)$ and all four SC-ES hybrids were statistically different to the parent cell lines $(P<0.001)$. However, to determine whether the increased copy number following fusion was the result of incomplete reprogramming or the tetraploid nature of the fusions, we analysed the mtDNA content of pluripotent ES-ES hybrid cells (ES-ES) [42]. They also had elevated levels of mtDNA copy number per cell $(259.76 \pm 6.32$; Fig. 1A), which were significantly different to levels in SC-ES 2 and $3(P<0.001)$, MEFs $(P<0.001)$, and the parental cell line, ESCs $(P<0.001)$.

To determine whether these outcomes resulted from discrete incomplete reprogramming of the somatic cells, we analysed the mtDNA copy number of three separate iPS cell lines (iPS 1,2 and 3) produced from the same MEF parental line used to generate SC-ES 1 to 4 . In their undifferentiated state, there was little variability between the lines with mean copy numbers per cell being $45.62 \pm$ 3.92, $54.12 \pm 6.47$ and $47.09 \pm 0.62$, respectively $(P>0.05$; Fig. $1 \mathrm{~A})$, which was not significantly different to the ESCs $(P>0.05$; Fig. $1 \mathrm{~A})$, but was significantly different to the parental cell line $(P<0.001)$. Finally, we examined undifferentiated NT-ES cells previously shown to be pluripotent and to contribute to all three germ layers [43]. This cell line contained elevated numbers of mtDNA copy $(196.93 \pm 13.96)$ compared to ESCs and iPS cells $(P<0.001)$ and similar copy number to SC-ES 1 and 4 cells and ES-ES cells (Fig. 1A). These data suggest that iPS cells derived from reprogrammed MEFs can mediate both pluripotent gene expression and mimic levels of mtDNA copy number 
associated with undifferentiated ESCs, whilst the cells derived from cell fusion and nuclear transfer fail to reset mtDNA copy number.

The relationship between mtDNA copy number and pluripotency

Using quantitative RT-PCR, we analysed the transcript levels of the pluripotent markers Oct4 (Fig. 1B), Nanog (Fig. 1C) and Sox2 (Fig. 1D) relative to ESCs in reprogrammed cells (iPS 2, SC-ES 1, ES-ES and NT-ES). Undifferentiated SC-ES 1 and ES-ES cells expressed similar levels of Oct4 to ESCs while transcripts were elevated in iPS $2(P<0.01)$ and reduced in NT-ES $(P<0.01)$ cells. Nanog transcripts in iPS and ES-ES cells were similar to ESCs levels, although SC-ES $(P<0.01)$ and NT-ES $(P<0.01)$ cells expressed relatively higher levels. Similarly, for Sox2 expression, SC-ES $(P<0.05)$ and NT-ES $(P<0.05)$ cells expressed significantly lower levels than observed for ESCs, whilst iPS and ESES cells were comparable to ESCs. The similarity in pluripotent gene expression between tetraploid ES-ES and ESCs most likely reflects the absence of nuclear reprogramming upon fusion of ESCs and the elevated mtDNA copy number in ES-ES cells may be attributed to their tetraploid nucleus. To further evaluate the effects of reprogramming on mtDNA copy number, we performed linear regression analysis of pluripotent gene expression ranked by statistical difference against mtDNA copy number (Fig. 1E). These calculations demonstrate their mtDNA copy number is positively and negatively correlated $\left(R^{2}=0.9039 ; P<0.05\right)$ with Nanog and Sox2, respectively. These correlations account for the elevated levels of mtDNA copy number observed in SC-ES and NT-ES cells.

\section{ATP content and OXPHOS complex expression in pluripotent stem cells}

Due to the differences in mtDNA copy number between the different reprogrammed cell types, we then examined the effects of reprogramming on ATP content (Fig. S1A). All reprogrammed pluripotent cell types and ESCs contained significantly lower levels of ATP than MEFs $(P<0.001)$. Although reprogramming by cell fusion (SC-ES and ES-ES) resulted in ATP levels being comparable to ESCs $(P>0.05)$, iPS cells had reduced cellular ATP compared to ESCs $(P<0.05)$. In order to determine whether the differences in copy number observed in reprogrammed cells influenced ATP content, we depleted reprogrammed somatic cells with the mitochondrial toxin, Rhodamine 6G (R6G; $1 \mu \mathrm{g} / \mathrm{ml}$ ), which is a known mtDNA depletion reagent $[46,47]$. After $72 \mathrm{hr}$ of $\mathrm{R} 6 \mathrm{G}$ treatment, mtDNA copy number of MEFs was depleted to $9.4 \%$ of control levels (Fig. S1B) and ATP content was reduced by 
33.7\% (Fig. S1A; P<0.05). Treatment of ESCs, and iPS 2, SC-ES 1 and ES-ES cells reduced mtDNA copy number to $26.5 \%, 27.6 \%, 8.7 \%$ and $9.9 \%$ of their untreated levels, respectively (Fig. S1B), though no significant effect was observed on ATP content (Fig. S1A). These data suggest that ESCs and reprogrammed cell types do not rely on OXPHOS as their main source of ATP.

In order to determine whether the lower ATP content observed in pluripotent cells was a consequence of low or absent OXPHOS complexes, we analysed the steady state levels of the OXPHOS complexes (Cl-IV) by BN-PAGE (Fig. S1C). CI, CII and CIV were present at similar levels in ESCs, iPS, SC-ES and NT-ES cells compared to MEFs, while ES-ES had elevated levels of CIII compared to ESCs and MEFs (Fig. S1C). These findings indicate that the low ATP content in pluripotent cells is not due to the absence of stable OXPHOS complexes.

\section{Expression of mtDNA replication factors in iPS and SC-ES cells}

In order to determine whether reprogrammed somatic cells are able to restrict mtDNA replication in their undifferentiated, pluripotent state, we labelled these cells with 5-bromo-2-deoxyuridine (BrdU) and the mitochondrial specific label, MitoTracker Red. iPS cells demonstrated increased incorporation of BrdU into their mtDNA when compared with ESC and SC-ES cells suggesting that they were more actively replicating mtDNA (Fig. 1F). We then investigated whether the differences in mtDNA copy number and BrdU incorporation in reprogrammed cell types were a consequence of variable expression of the mtDNA-specific replication factors. We analysed each of the cell lines for expression of Tfam, Twinkle (Peo1), mtSsbp1, PolgA and PolgB mRNA by real time PCR (qPCR) (Fig. S2). For PolgA, only ES-ES cells $(\mathrm{P}>0.05)$ were not statistically different to undifferentiated ESCs (Fig. S2A). The MEF cells expressed statistically less PolgA $(\mathrm{P}<0.001)$ than observed for undifferentiated ESCs, while the SC-ES and iPS cells expressed significantly more $(P<0.001$; see Fig. S2A). Peo1 levels in MEFs and SC-ES 1, 2 and 4 cells were, however, only significantly different to ESCs (Fig. S2B). When compared to ESCs, SC-ES 2 and iPS 1 and 2 cells expressed significantly less $m t S s b p 1$ (Fig. S2C). PolgB levels were significantly reduced in ES-ES cells ( $P<0.01)$, iPS 1,2 and 3 cells $(P<0.01)$, MEFs $(P<0.001)$ and SC-ES 2 and 4 cells $(P<0.01)$ whilst SC-ES 1 cells expressed significantly more $(\mathrm{P}<0.01$; Fig. S2D). Furthermore, MEF levels were considerably reduced when compared to all cell types $(\mathrm{P}<0.001)$. The patterns of expression for Tfam demonstrated little 
difference between all pluripotent cell types (Fig. S2E), however, they all expressed elevated levels when compared to MEFs $(\mathrm{P}<0.001)$.

\section{Expression of genes of differentiation in reprogrammed cells}

We verified the potential of the reprogrammed cell types to differentiate into all three germ layers (Fig. 2). Each reprogrammed cell type expressed genes characteristic of the mesoderm (CD31 and VEGFR), ectoderm (Nestin and MAP2) and endoderm (AFP and FoxA2). Nevertheless, we observed some differences between cell types. Of note, MAP2 expression was lower in NT-ES cells prior to Day 14 and FoxA2 expression peaked earlier in SC-ES cells than in other cell types (Fig. 2).

\section{Patterns of mtDNA replication in differentiating reprogrammed cells}

During the early stages of ESC differentiation, mtDNA replication remains strictly regulated with a key replication event taking place on Day 6 of differentiation resulting in a significant increase in the number of mtDNA copies/cell [14]. To assess whether reprogrammed cells were able to regulate their mtDNA copy number in a manner similar to ESCs during the process of differentiation, we generated embryoid bodies and analysed their mtDNA replication events at $24 \mathrm{hr}$ intervals during Days 1 to 7 and Day 14 of differentiation. Firstly, we focused on mtDNA copy number during differentiation. After an initial increase in mtDNA copy number in SC-ES 1 cells at $24 \mathrm{hr}$ of differentiation $(P<0.001)$, levels dropped to lower than those of the undifferentiated cells on Days 2,4 and $5(P<0.001)$ whilst levels were not significantly different on Days 3, 6 and 7 (P>0.05) (Fig. 3A). We then interrogated iPS 2 cells and detected a completely different pattern (Fig. 3B), where following an initial burst of mtDNA replication on Day 1, mtDNA copy number returned to steady state levels on Day 2. However, there were significant increases on Days 3 and $5(P<0.001)$, followed by a return to low levels of copy on Days 4 and 6. Consequently, neither cell type exhibited the large increase in mtDNA copy number observed in ESCs on Day 6 [14]. Whilst both sets of reprogrammed lines had approximately 200 copies of mtDNA per cell on Day 7, there were significantly more copies present on Day 14 for the hybrid cells $(P<0.001)$, as would be anticipated in cells committing to various fates. Again dualstaining with BrdU and MitoTracker confirmed that mtDNA replication was active during the early stages of differentiation (Fig. 3C) and that SC-ES 1 and iPS 2 cells had extended cytoplasms. 
In order to determine whether discordant mtDNA replication events during differentiation were a consequence of nuclear reprogramming or ploidy, we analysed mtDNA copy number for ES-ES cell fusions and NT-ES cells. After 24 hr of differentiation, no difference was detected for ES-ES cells (Fig. 3D), whilst NT-ES cells (Fig. 3E) displayed a significant increase $(P<0.001)$, similar to events reported for R1 ESCs [14], but at far lower levels. On Days 6 and 7 of ES-ES differentiation, a progressive increase in copy number was evident, with a considerable increase by Day $14(P<0.001)$. At the same time points, NT-ES cells had a copy number similar to their undifferentiated cells (Fig. 3E).

We then determined if mtDNA turn over on Day 6 of differentiation was associated with the loss of pluripotency, specifically the levels of expression of Oct 4, Nanog and Sox2 on Days 6 and 7 of differentiation. On Day 6 of differentiation, Oct4 transcript levels were reduced compared to undifferentiated ESCs for all cell types (Fig. 1B) and no differences were observed between the cell types. Similar levels of Oct4 transcript were observed on Day 7 although the ES-ES cell line demonstrated a further reduction between Days 6 and 7 (Fig. 1B; $\mathrm{P}<0.001$ ). Nanog expression for ESCs remained statistically similar throughout differentiation although iPS cells expressed high levels on Day $6(P<0.01)$ and extremely low levels on Day $7(P<0.01$; Fig. $1 C)$. SC-ES $(P<0.01)$ and NT-ES $(\mathrm{P}<0.001)$ cells contained fewer Nanog transcripts on Day 6 compared to their undifferentiated states, and these levels were similar to those observed for ESCs. Expression of Sox2 in iPS cells was reduced on Days 6 and 7 of differentiation compared to the undifferentiated state, whilst ES-ES cells demonstrated a decrease on day $7(P<0.01$; Fig. 1D). Both SC-ES $(P<0.01)$ and NT-ES $(P<0.01)$ cells expressed higher levels of Sox2 on Day 7 compared to their undifferentiated counterparts and ESCs. Linear regression analysis of mtDNA copy number and pluripotent gene expression determined that mtDNA copy number was not a function of pluripotent gene expression during differentiation (data not shown).

\section{Expression of mtDNA replication factors during differentiation of pluripotent stem cells}

As BrdU staining and variability in mtDNA copy number indicated that diverse mtDNA replication events were taking place during differentiation, we determined the levels of transcriptional activity for Tfam (Fig. 4A), Peo1 (Fig. 4B), mtSsbp1 (Fig. 4C), PolgA (Fig. 4D) and PolgB (Fig. 4E) at several key stages of mtDNA replication during differentiation. It was evident that there were considerably lower 
levels of expression for Peo1 on Days 1, 7 and 14 in the reprogrammed somatic cells when compared to ESCs at the same time point (Fig. 4B; P<0.01 to 0.001). There was significantly less Tfam (Fig. 4A) and PolgA (Fig. 4D) on Days 1, 7 and 14 when compared to their undifferentiated counterparts. However, the increased levels of PolgA (Fig. 4D) in SC-ES 1 cells on Days 7 and 14, compared to Day 1 , suggest that they were primed for mtDNA replication.

\section{ATP content and the steady state levels of the OXPHOS complexes during differentiation}

We then determined whether the disparities in mtDNA copy number and mtDNA replication factor expression in the reprogrammed somatic cells during differentiation would affect their capacity to produce ATP during differentiation. We observed on Day 7 of differentiation that iPS cells contained significantly less ATP than ESCs $(P<0.01)$ whilst SC-ES had elevated levels of ATP $(P<0.05$; Fig. S3A). Subsequently, ATP levels on Day 14 were not significantly different between ESC, iPS, SC-ES and ES-ES cells ( $P>0.05$; Fig. S3B) though these levels were statistically elevated compared to their undifferentiated counterparts $(P<0.001$ : Fig. S1A cf Fig. S3B).

Analysis of steady state levels for the OXPHOS complexes after resolving by BN-PAGE revealed that iPS cells, on day 7 of differentiation, contained higher levels of $\mathrm{CI}$, CIII and CIV than ESCs and MEFs (Fig. S3C). At the same time point, ESCs, ES-ES and NT-ES cells all contained similar levels of CI-IV whilst SC-ES cells contained lower steady state levels of CIV compared to ESCs (Fig. S3C).

\section{The effects of 5-azacytidine (5-Aza) on mtDNA replication and copy number}

As it has been previously shown that treatment of low-passage iPS cells with chromatin modifying drugs improves reprogramming outcomes $[32,38,39]$, we hypothesized that resetting the epigenome of ESCs and reprogrammed somatic cells with the DNA methyltransferase inhibitor, 5-Azacytidine (5Aza), would directly influence mtDNA copy number. Firstly, we treated ESCs with 5-Aza (ESC $\left.{ }^{5-A z a}\right)$ for 72 hrs prior to embryoid body formation and differentiation. In undifferentiated cells, this increased ESC $^{5-A z a}$ mtDNA copy number from $36.56 \pm 1.79$ to $61.23 \pm 5.59$ (Fig. 5A), whilst 5-Aza treatment had no effect on iPS (iPS ${ }^{5-A z a}$ ) cell mtDNA copy number ( $P>0.05$; Fig. 5B) but resulted in an increase in ATP levels in iPS cells, similar to ESCs $(\mathrm{P}<0.05$; Fig. 5F). Following 6 days of differentiation, ESC mtDNA copy number decreased from 2370 to $420.92 \pm 53.65$ with a similar pattern being observed on 
Day 14 (Fig. 5A). On the other hand, iPS ${ }^{5-A z a}$ cells were able to mediate the significant increase in mtDNA copy number on Day $6(2112.82 \pm 111.53)$ and Day $14(7228.45 \pm 141.97)$ with a concurrent reduction observed on Day 7 (579.15 \pm 43.87 ; Fig. 5B cf Fig. 3B). Conversely, undifferentiated SCES $^{5-A z a}$ (Fig 5C cf Fig. 3C) and ES-ES ${ }^{5-A z a}$ (Fig. 5D cf Fig. 3D) cells contained significantly fewer copies of mtDNA (195.79 $\pm 13.87 ; \mathrm{P}<0.05$ and $186.96 \pm 6.03$, respectively; $\mathrm{P}<0.001)$ after 5 -Aza treatment. SC-ES ${ }^{5-A z a}$ (Fig. 5C) cells were unable to increase mtDNA copy on Day 6 (876.91 \pm $101.49)$ and $14(883.8 \pm 79.94)$ to match ESCs though numbers were increased when compared to their untreated counterparts (Fig. 3A). In ES-ES ${ }^{5-A z a}$ cells, an increase to $1896.37 \pm 128.79$ and a decrease to $363.31 \pm 12.78 \mathrm{mtDNA}$ copies/cell were also detected on Days 6 and 7 , respectively. This was followed by an increase on Day 14 (1182.82 \pm 47.74 mtDNA copies/cell; Fig. 5D), which, although dissimilar to ESCs, resulted in a different mtDNA copy number profile during differentiation when compared to untreated cells (Fig 3D). Treatment of NT-ES cells with 5-Aza (NT-ES ${ }^{5-A z a}$; Fig 5E cf Fig. 3E) had no effect on the undifferentiated state (203.23 \pm 17.32 mtDNA copies/cell). However, during differentiation, mtDNA copy number decreased on Day $1(158.82 \pm 17.81)$ and significantly increased on Day $7(1155.12 \pm 137.28)$. The outcomes show that treatment with 5 Aza results in iPS, but not SC-ES and NT-ES, cells being able to mediate their mtDNA replication events in a manner similar to ESCs. 


\section{Discussion}

The data presented here demonstrate that somatic cell reprogramming by cell fusion (SC-ES cells) and NT results in a 5- to 9-fold higher mtDNA copy number per pluripotent stem cell than observed in ESCs, whilst undifferentiated iPS cells have similar mtDNA copy number to ESCs. Nevertheless, none of the reprogrammed cell types were able to modulate the key mtDNA replication events that take place in ESCs during differentiation. However, the culture of undifferentiated reprogrammed cells in 5Aza demonstrated that iPS cells, but not SC-ES and NT-ES cells, could mimic the key mtDNA replication events of the ESCs during differentiation.

We have previously hypothesised that pluripotent stem cells establish the 'mtDNA set point' $[19,20]$, which ensures that they maintain low numbers of mtDNA during pluripotency and early differentiation. In this instance, mtDNA replication is restricted to replenishing the mitochondrial genome following cell division or when cells reach precariously low levels of mtDNA. ESCs maintain this pattern during the early stages of differentiation except for one major replication event that takes place on Day 6 of differentiation. MtDNA copy number then returns to low levels until Day 11 [14, 21]. Consequently, the establishment of the mtDNA set point is an essential part of cellular differentiation as it enables differentiating cells to acquire the appropriate numbers of mtDNA to meet the OXPHOS specific requirements of the specialised cell and its function once commitment has taken place [21].

Our data suggest that the establishment of the mtDNA set point is dependent on the degree of pluripotency within cells. The elevated mtDNA copy number observed in undifferentiated SC-ES and NT-ES cells correlated with the variable levels of expression of the pluripotent transcription factors, Sox2 and Nanog, whereby they exhibited elevated levels of Nanog and decreased levels of Sox2 when compared with ESCs. Furthermore, Oct4 expression was reduced in NT-ES cells, while iPS cells displayed elevated Oct4 expression and low copy number. Whilst upregulation of Nanog has been proposed as an essential requirement for establishing and maintaining pluripotency $[48,49]$, variation within the OCT4-SOX2-NANOG network alters the expression of their target genes, including their own expression and triggers the onset of differentiation $[50,51,52]$. We argue that the disparity in expression of the genes of the OCT4-SOX2-NANOG network could be attributed to incomplete reprogramming of the somatic genome, as described by others [32,53] resulting in the failure to fully 
establish pluripotency and thus the mtDNA set point. Indeed, undifferentiated iPS cells were also primed for mtDNA replication, as demonstrated by increased levels of labelling for BrdU coupled to the increased expression of PolgA. Consequently, reduced mitochondrial expansion and mtDNA copy number may be attributed to mitochondrial regulatory processes, such as mitophagy, as observed in ESCs [53]. Nevertheless, iPS cells appear to be unable to exert strict control on mtDNA replication, hence the 2 to 3 fold increase in mtDNA copy number and expanded cytoplasms observed on Day 1 of differentiation. Although undifferentiated ES-ES fusions displayed similar levels of expression for Oct4, Nanog and Sox2 to ESCs, they have elevated mtDNA copies. This suggests that the regulation of mtDNA copy number in ES-ES cells is influenced by their tetraploid nature. In the case of the undifferentiated SC-ES cells, their partial reprogramming and tetraploidy is likely to further complicate their ability to regulate mtDNA copy number.

The copy number present in the SC-ES and NT-ES cells is very similar to somatic progenitor cells, which tend to have approximately 200 copies of mtDNA per cell [54], as do primordial germ cells (PGCs) [55]. In terms of their mtDNA profiles, SC-ES and NT-ES cells are possibly further down the pathway of differentiation than ESCs. Nevertheless, this phenomenon is not unique as PGCs reexpress key pluripotent genes during differentiation [56] as do some cancer stem cells [57], suggesting that reestablishment of pluripotent gene expression does not necessarily reduce mtDNA copy number to mtDNA set point levels. Not only do PGCs re-establish pluripotency, they express other cell specific transcriptional regulators $[58,59,60]$, which, as for other progenitor cells, will ensure that mtDNA copy number is tightly controlled. However, variations of this process do not seem to have been acquired by reprogrammed somatic cells.

In support of our hypothesis that incomplete reprogramming fails to establish the mtDNA set point, we have observed, as have others [61], that the expression of pluripotent genes, such as Oct4, Nanog, Dppa5, Pramel7 and Ndp52116 [14,21], fluctuate as differentiation is initiated. The patterns of pluripotent gene expression we observed in the undifferentiated reprogrammed somatic cells is similar. Consequently, none of the reprogrammed somatic cell types, were able to maintain the low numbers of mtDNA copy during the early stages of differentiation, as demonstrated by differentiating ESCs. Most critically, none of the reprogrammed cell types were able to mediate the key mtDNA 
replication event that takes place on Day 6 of differentiation, and exhibited quite distinct patterns of gene expression for Nanog and Sox2 on Days 6 and 7. However, it appears that iPS cells attempt to compensate through a small increase in mtDNA copy number on Day 7 , though they also failed to mediate the increase in mtDNA copy number on Day 11. Therefore, we propose that, by not having clearly established pluripotency, reprogrammed somatic cells do not establish the 'mtDNA set point', which further reflects their inability to coordinate the assembly of the electron transfer chain, as shown on Day 7 in iPS cells.

The effects of reprogramming are likely to directly influence mtDNA replication, as this process is highly dependent on the expression of nuclear-encoded genes that, once transcribed and translated, translocate to the mitochondrion. Indeed, the mitochondrial-specific polymerase, PolgA, appears to be an important sensor for both pluripotency and mtDNA proliferation during early development. It has previously been shown that partial knockdown of PolgA in undifferentiated ESCs results in the decrease in Oct4 expression and forces differentiation into early lineages [14]. The failure to initiate mtDNA replication through the expression of PolgA at specific stages during early development has undesired effects whereby PolgA ${ }^{+-}$mice exhibit mtDNA depletion syndromes, which result in serious effects on organ function, and, in particular, triggers the onset of cardiomyopathy [18]. On the other hand, PolgA ${ }^{-/}$mice are embryonic lethal from E7.5, a time point that is comparable to Day 6 of ESC differentiation [17]. Furthermore, $\mathrm{Tfam}^{+-}$mice also exhibit mtDNA depletion and $\mathrm{Tfam}^{-/}$mouse embryos do not survive after E8.5 to 10.5. [62], which is equivalent to Day 7+ of differentiation. Consequently, it is likely that failure to mediate the key mtDNA turnover event at Day 6 would result in differentiated cells possessing few copies of mtDNA, and thus exhibit mtDNA depletion-like syndromes $[63,64]$.

Whilst the differences in the regulation of mtDNA copy number might be an indirect effect of cellular ploidy in SC-ES and ES-ES cells, thus altering the expression of the nuclear-encoded mtDNA replication factors and, in turn, mtDNA copy number, the observed outcomes are likely to arise from aberrant epigenetic regulation. Indeed, recent data demonstrate that reprogrammed cells are not equivalent to ESCs $[35,38,39,65,66]$. ESC equivalence is acquired in iPS cells when cultured continually [26, 29, 31], serially reprogrammed [26] or treated with epigenetic modifying agents 
$[32,38,39]$. The treatment of partially reprogrammed iPS cells with 5-Aza [32,39] and valproic acid [38] induces epigenetic changes that improve the reprogramming outcomes of iPS cells. In agreement with these studies, we find differential expression of genes involved in mitochondrial biogenesis (PolgA and Peo1) characteristic of differentiated cells, and a consequent failure to accumulate thousands of mtDNA copies per cell. The alterations in mtDNA copy number, and ATP generation, in 5-Aza treated iPS cells validates the above conclusions and suggests that the regulation of mtDNA replication may be either directly or indirectly related to the epigenetic regulation of one or more of the nuclearencoded mtDNA-specific replication factors or the downstream effects of general reprogramming.

The ability of a cell to regulate mtDNA replication effectively is perhaps inherent of it having undergone a consistent process of regulation. As ESCs are derived from the ICM cells of the blastocyst [73], they will have undergone a process of mtDNA reduction mediated by the preimplantation embryo with each newly divided cell having fewer copies of mtDNA [12]. Although mtDNA replication is triggered at the blastocyst stage in the trophectoderm, the $4.83 \times 10^{3} \mathrm{mtDNA}$ copies present in the ICM cells continue to be reduced until the onset of gastrulation [13], as demonstrated by 30 and 45 copies in mouse ESCs $[14,21]$. It is this continual reduction in mtDNA copy number that establishes the mtDNA set point, which is maintained during the early stages of differentiation. This allows cells during the later stages of differentiation to acquire the appropriate numbers of mtDNA to support their specific OXPHOS-derived ATP requirements that are suited to their specialisation [21]. Our data would indicate that none of the reprogrammed somatic cells have acquired the mtDNA set point as evidenced by their failure to reduce their mtDNA copy number in the undifferentiated state (SC-ES and NT-ES cells) and maintain this state during the early stages of differentiation (SC-ES, NT-ES and iPS cells).

The differential regulation of mtDNA replication in differentiating reprogrammed cells would probably generate outcomes similar to those associated with NT where mtDNA replication is not controlled in the preimplantation embryo with persistent upregulation of the mtDNA replication factors $[16,30]$. Many of these offspring do not survive post-parturition because, once ex-utero, they are no longer able to support themselves metabolically [67]. Our data suggest that ESCs derived from NT embryos display aberrant reprogramming during in vitro differentiation consistent with the inefficient production of 
cloned offspring. However, recent reports suggest NT-ES cells are phenotypically similar to fertilised ESCs [32]. These differences may be attributed to the use of a MEK1 inhibitor during NT-ESC derivation [27,32,49,52], which promotes reprogramming and establishment of ESCs $[49,52,76,68]$. This failure of NT-ES cells to regulate mtDNA copy during differentiation suggests that the influence of control on mtDNA copy number may arise from the maturing oocyte, which modifies its mtDNA content up to the metaphase II stage, so that under normal conditions mtDNA replication is restricted and species-specific changes in mtDNA copy number occur during preimplantation development [11]. Consequently, any reprogrammed somatic cell will have escaped these effects and, in combination with partial modulation of the mtDNA replication factors, a nucleus transferred into an enucleated oocyte will elicit aberrant patterns of mtDNA replication providing further distortion to early mtDNA replication events.

Previous reports of human pluripotent cells have demonstrated a similarity in mtDNA copy number between differentiated ESCs and iPS cells $[69,70]$. In this context, the deregulation of mtDNA copy number presented here may be attributed to variations between human and mouse mtDNA turn over during differentiation $[14,71]$ or the heterogeneous nature of the reprogramming process $[32,33,34,52]$. The variability in mtDNA copy number during the early stages of differentiation impacts on the ability of reprogrammed cells to generate appropriate levels of ATP at these stages, as demonstrated by iPS cells containing significantly less ATP and SC-ES cells producing significantly more on Day 7 than ESCs. Contrastingly, on day 7 of iPS differentiation the steady state levels of $\mathrm{Cl}$, III and IV were considerably higher than all other cell types analysed. The levels of ATP generated on Day 14 and levels of steady state complexes for iPS and SC-ES were similar to ESCs while mtDNA copy number was considerably lower. These discrepancies between ATP and mtDNA copy number in iPS cells may reflect a compensatory mechanism whereby expression of the OXPHOS subunits is increased to counteract low levels of ATP. Indeed, increased subunit expression is observed in some mitochondrial-type diseases to compensate for defects in the ETC $[72,73]$. However, the inability of reprogrammed cells to produce mtDNA copy number characteristic of differentiated cells was not reflected in the levels of ETC complexes or the ATP content on day 14. The lack of correlation between ATP content and mtDNA copy number during later stages of differentiation mimics mtDNA disease phenotypes in which debilitating mutations in the mtDNA manifest as clinical symptoms only 
once a threshold of mutant vs wild-type is breached in a stage specific manner [74]. In some instances, mutant threshold correlates with reduced OXPHOS capacity only upon high energy demand, for example during exercise [75].

The importance of the continued upregulation of mtDNA copy number to the generation of ATP has been demonstrated in cybrid models of mtDNA disease [76]. These anomalies are of concern as there is a number of ATP-mediated developmental events that take place during these early stages. These include the epigenetic regulation of gene expression [77], which will impact on cellular differentiation and function with the likelihood that ATP levels determine the extent to which these epigenetic modifications can take place [78]. It has been accepted that the mitochondrial genome is not methylated [79,80], although a recent study has reopened this debate [81]. Since the mtDNA replication factors are nuclear encoded, it is likely that ATP-mediated epigenetic regulation of mtDNA will function through nuclear factors. There is some evidence suggesting that the expression of PolgA is mediated by epigenetic modification, namely an increase in DNA methylation of exon-2 of this gene [82]. Such regulation of mtDNA appears to be vital during spermatogenesis as those sperm cells capable of fertilisation have significantly lower numbers of mtDNA copy compared to those that fail to fertilise [82]. This mechanism would also account for the cell specific regulation of mtDNA copy number where there is large variability between cell types [15].

In conclusion, we propose that the control of mtDNA replication following somatic cell reprogramming is dependent on the complete reestablishment of pluripotency including the OCT4-SOX2-NANOG network. This would enable the mtDNA set point to be established so that mtDNA copy number is strictly regulated during pluripotency and differentiation. It is apparent from our data that this process is better achieved by cells undergoing induced pluripotency, since they adopt mtDNA replication and ATP profiles similar to ESCs during pluripotency and differentiation, following treatment with an epigenetic modifier. Nevertheless, the epigenetic control of mtDNA replication still requires elucidation, which is most likely a phenomenon that is inherited or set in the oocyte just prior to fertilisation. 


\section{Acknowledgements}

This work is supported by funding from the British Heart Foundation (PG/04/117) and the Medical Research Council, UK (grant number: G0600273) and the Victorian Government's Operational Inftrastructure Support Program. We are grateful to Dr Megan Munsie, Australian Stem Cell Centre, for the somatic cell nuclear transfer embryonic stem cell line (NT-ES); and Ms Jacqui Johnson, Centre for Reproduction and Development, Monash Institute of Medical Research for expertise in stem cell culture. 


\section{References}

1. Anderson, S., Bankier, A.T., Barrell, B.G., de Bruijn, M.H., Coulson, A.R., et al. (1981) Sequence and organization of the human mitochondrial genome. Nature, 290, 457-465.

2. Pfeiffer, T., Schuster, S., Bonhoeffer, S., (2001) Cooperation and competition in the evolution of ATP-producing pathways. Science, 292, 504-507.

3. Fernandez-Vizarra, E., Tiranti, V., Zeviani, M., (2009) Assembly of the oxidative phosphorylation system in humans: what we have learned by studying its defects. Biochimica Biophys Acta, 1793, 200-211.

4. Lazarou, M., Thorburn, D.R., Ryan, M.T., McKenzie, M., (2009) Assembly of mitochondrial complex I and defects in disease. Biochim Biophys Acta, 1793, 78-88.

5. McKenzie, M., Ryan, M.T., (2010) Assembly factors of human mitochondrial complex I and their defects in disease. IUBMB Life, 62, 497-502.

6. Wallace, D.C., (1999) Mitochondrial diseases in man and mouse. Science 283: 1482-1488.

7. Smeitink J, van den Heuvel L, DiMauro S (2001) The genetics and pathology of oxidative phosphorylation. Nature Review Genetics, 2, 342-352.

8. $\quad$ Clayton, D.A., (1982) Replication of animal mitochondrial DNA. Cell 28: 693-705.

9. Korhonen, J.A., Gaspari, M., Falkenberg, M., (2003) TWINKLE Has 5' -> 3' DNA helicase activity and is specifically stimulated by mitochondrial single-stranded DNA-binding protein. Journal Biological Chemistry, 278, 48627-48632.

10. Lazarou, M., McKenzie, M., Ohtake, A., Thorburn, D.R., Ryan, M.T., (2007) Analysis of the assembly profiles for mitochondrial- and nuclear-DNA-encoded subunits into complex I. Molecular and Cellular Biology, 27, 4228-4237.

11. Spikings, E.C., Alderson, J., St John, J.C., (2007) Regulated mitochondrial DNA replication during oocyte maturation is essential for successful porcine embryonic development. Biology of Reproduction, 76, 327-335.

12. Thundathil, J., Filion, F., Smith, L.C., (2005) Molecular control of mitochondrial function in preimplantation mouse embryos. Molecular Reproduction and Development, 71, 405-413.

13. Cao, L., Shitara, H., Horii, T., Nagao, Y., Imai, H., et al. (2007) The mitochondrial bottleneck occurs without reduction of mtDNA content in female mouse germ cells. Nature Genetic, 39, 386-390.

14. Facucho-Oliveira, J.M., Alderson, J., Spikings, E.C., Egginton, S., St John, J.C., (2007) Mitochondrial DNA replication during differentiation of murine embryonic stem cells. Journal of Cell Science, 120, 4025-4034.

15. Miller, F.J., Rosenfeldt, F.L., Zhang, C., Linnane, A.W., Nagley, P., (2003) Precise determination of mitochondrial DNA copy number in human skeletal and cardiac muscle by a PCR-based assay: lack of change of copy number with age. Nucleic Acids Research, 31, e61.

16. Bowles, E.J., Lee, J.H., Alberio, R., Lloyd, R.E., Stekel, D., et al. (2007) Contrasting effects of in vitro fertilization and nuclear transfer on the expression of mtDNA replication factors. Genetics, 176, 1511-1526.

17. Leahy, A., Xiong, J.W., Kuhnert, F., Stuhlmann, H., (1999) Use of developmental marker genes to define temporal and spatial patterns of differentiation during embryoid body formation. Journal of Experimental Zoology, 284, 67-81.

18. Hance, N., Ekstrand, M.I., Trifunovic, A., (2005) Mitochondrial DNA polymerase gamma is essential for mammalian embryogenesis. Human Molecular Genetics, 14, 1775-1783.

19. St John, J.C., Facucho-Oliveira, J., Jiang, Y., Kelly, R., Salah, R., (2010) Mitochondrial DNA transmission, replication and inheritance: a journey from the gamete through the embryo and into offspring and embryonic stem cells. Human Reproductive Update, 16, 488-509.

20. Kelly, R.D., St John, J.C., (2010) Role of mitochondrial DNA replication during differentiation of reprogrammed stem cells. International Journal of Developmental Biology, 54, 1659-1670

21. Facucho-Oliveira, J.M., St John, J.C., (2009) The relationship between pluripotency and mitochondrial DNA proliferation during early embryo development and embryonic stem cell differentiation. Stem Cell Reviews and Reports, 5, 140-158.

22. Cowan, C.A., Atienza, J., Melton, D.A., Eggan, K., (2005) Nuclear reprogramming of somatic cells after fusion with human embryonic stem cells. Science, 309, 1369-1373.

23. Yu, J., Vodyanik, M.A., He, P., Slukvin, I.I., Thomson, J.A., (2006) Human embryonic stem cells reprogram myeloid precursors following cell-cell fusion. Stem Cells, 24, 168-176. 
24. Takahashi, K., Yamanaka, S., (2006) Induction of pluripotent stem cells from mouse embryonic and adult fibroblast cultures by defined factors. Cell, 126, 663-676.

25. Takahashi, K., Tanabe, K., Ohnuki, M., Narita, M., Ichisaka, T., et al. (2007) Induction of pluripotent stem cells from adult human fibroblasts by defined factors. Cell, 131, 861-872.

26. Wilmut, I., Schnieke, A.E., McWhir, J., Kind, A.J., Campbell, K.H., (1997) Viable offspring derived from fetal and adult mammalian cells. Nature, 385, 810-813.

27. Blelloch, R., Wang, Z., Meissner, A., Pollard, S., Smith, A., et al. (2006) Reprogramming efficiency following somatic cell nuclear transfer is influenced by the differentiation and methylation state of the donor nucleus. Stem Cells, 24, 2007-2013.

28. Dean, W., Santos, F., Stojkovic, M., Zakhartchenko, V., Walter, J., et al. (2001) Conservation of methylation reprogramming in mammalian development: aberrant reprogramming in cloned embryos. Proceedings of the National Academy of Science USA, 98, 13734-13738.

29. Gurdon, J.B., Melton, D.A., (2008) Nuclear reprogramming in cells. Science, 322, 1811-1815.

30. Lloyd, R.E., Lee, J.H., Alberio, R., Bowles, E.J., Ramalho-Santos, J., et al. (2006) Aberrant nucleo-cytoplasmic cross-talk results in donor cell mtDNA persistence in cloned embryos. Genetics, 172, 2515-2527.

31. Brambrink, T., Hochedlinger, K., Bell, G., Jaenisch, R., (2006) ES cells derived from cloned and fertilized blastocysts are transcriptionally and functionally indistinguishable. Proceedings of the National Academy of Science USA, 103, 933-938.

32. Kim K, Doi A, Wen B, Ng K, Zhao R, et al. (2010) Epigenetic memory in induced pluripotent stem cells. Nature 467: 285-290.

33. Feng, Q., Lu, S.J., Klimanskaya, I., Gomes, I., Kim, D., et al. (2010) Hemangioblastic derivatives from human induced pluripotent stem cells exhibit limited expansion and early senescence. Stem Cells, 28, 704-712.

34. Hu, B.Y., Weick, J.P., Yu, J., Ma, L.X., Zhang, X.Q., et al. (2010) Neural differentiation of human induced pluripotent stem cells follows developmental principles but with variable potency. Proceedings of the National Academy of Science USA, 107, 4335-4340.

35. Chin, M.H., Mason, M.J., Xie, W., Volinia, S., Singer, M., et al. (2009) Induced pluripotent stem cells and embryonic stem cells are distinguished by gene expression signatures. Cell Stem Cell, 5, 111-123.

36. Hanna, J., Saha, K., Pando, B., van Zon, J., Lengner, C.J., et al. (2009) Direct cell reprogramming is a stochastic process amenable to acceleration. Nature, 462, 595-601.

37. Polo, J.M., Liu, S., Figueroa, M.E., Kulalert, W., Eminli, S., et al. (2010) Cell type of origin influences the molecular and functional properties of mouse induced pluripotent stem cells. Nature Biotechnology, 28, 848-855.

38. Stadtfeld, M., Apostolou, E., Akutsu, H., Fukuda, A., Follett, P., et al. (2010) Aberrant silencing of imprinted genes on chromosome $12 \mathrm{qF} 1$ in mouse induced pluripotent stem cells. Nature, 465, 175-181.

39. Mikkelsen, T.S., Hanna, J., Zhang, X., Ku, M., Wernig, M., et al. (2008) Dissecting direct reprogramming through integrative genomic analysis. Nature, 454, 49-55.

40. Sumer, H., Jones, K.L., Liu, J., Heffernan, C., Tat, P.A., et al. (2009) Reprogramming of somatic cells after fusion with iPS and ntES cells. Stem Cells and Development, 19, 239-246.

41. Sumer, H., Nicholls, C., Pinto, A.R., Indraharan, D., Liu, J., et al. (2010) Chromosomal and telomeric reprogramming following ES-somatic cell fusion. Chromosoma, 119, 167-176.

42. Sumer, H., Jones, K.L., Liu, J., Rollo, B.N., van Boxtel, A.L., et al. (2009) Transcriptional changes in somatic cells recovered from embryonic stem-somatic heterokaryons. Stem Cells and Development, 18, 1361-1368.

43. Munsie, M.J., Michalska, A.E., O'Brien, C.M., Trounson, A.O., Pera, M.F., et al. (2000) Isolation of pluripotent embryonic stem cells from reprogrammed adult mouse somatic cell nuclei. Current Biology, 10, 989-992.

44. Keller, G.M., (1995) In vitro differentiation of embryonic stem cells. Current Opinions in Cell Biology, 7, 862-869.

45. McKenzie, M., Lazarou, M., Ryan, M.T., (2009) Chapter 18 Analysis of respiratory chain complex assembly with radiolabeled nuclear- and mitochondrial-encoded subunits. Methods in Enzymology, 456, 321-339.

46. McKenzie, M., Trounce, I.A., Cassar, C.A., Pinkert, C.A., (2004) Production of homoplasmic xenomitochondrial mice. Proceedings of the National Academy of Science USA, 101, 16851690.

47. Trounce, I., Wallace, D.C., (1996) Production of transmitochondrial mouse cell lines by cybrid rescue of rhodamine-6G pre-treated L-cells. Somatic Cell and Molecular Genetics, 22, 81-85. 
48. Silva, J., Chambers, I., Pollard, S., Smith, A., (2006) Nanog promotes transfer of pluripotency after cell fusion. Nature, 441, 997-1001.

49. Silva, J., Nichols, J., Theunissen, T.W., Guo, G., van Oosten, A.L., et al. (2009) Nanog is the gateway to the pluripotent ground state. Cell, 138, 722-737.

50. Niwa, H., Miyazaki, J., Smith, A.G., (2000) Quantitative expression of Oct-3/4 defines differentiation, dedifferentiation or self-renewal of ES cells. Nature Genetics, 24, 372-376.

51. Chen, X., Xu, H., Yuan, P., Fang, F., Huss, M., et al. (2008) Integration of external signaling pathways with the core transcriptional network in embryonic stem cells. Cell, 133, 1106-1117.

52. Hanna, J.H., Saha, K., Jaenisch, R., (2010) Pluripotency and cellular reprogramming: facts, hypotheses, unresolved issues. Cell, 143, 508-525.

53. Todd, L.R., Damin, M.N., Gomathinayagam, R., Horn, S.R., Means, A.R., et al. (2010) Growth factor erv1-like modulates Drp1 to preserve mitochondrial dynamics and function in mouse embryonic stem cells. Molecular Biology of the Cell, 21, 1225-1236.

54. Rae, P.C., Kelly, R.D., Egginton, S., St John, J.C., (2011) Angiogenic potential of endothelial progenitor cells and embryonic stem cells. Vascular Cell, $3,11$.

55. Wai, T., Teoli, D., Shoubridge, E.A., (2008) The mitochondrial DNA genetic bottleneck results from replication of a subpopulation of genomes. Nature Genetics, 40, 1484-1488.

56. Surani, M.A., Durcova-Hills, G., Hajkova, P., Hayashi, K., Tee, W.W., (2008) Germ line, stem cells, and epigenetic reprogramming. Cold Spring Harbour Symposia on Quantative Biology, 73, 9-15.

57. Kang, J., Shakya, A., Tantin, D., (2009) Stem cells, stress, metabolism and cancer: a drama in two Octs. Trends in Biochemical Sciences, 34, 491-499.

58. Chambers, I., Silva, J., Colby, D., Nichols, J., Nijmeijer, B., et al. (2007) Nanog safeguards pluripotency and mediates germline development. Nature, 450, 1230-1234.

59. Maldonado-Saldivia, J., van den Bergen, J., Krouskos, M., Gilchrist, M., Lee, C., et al. (2007) Dppa2 and Dppa4 are closely linked SAP motif genes restricted to pluripotent cells and the germ line. Stem Cells, 25, 19-28.

60. Kehler, J., Tolkunova, E., Koschorz, B., Pesce, M., Gentile, L., et al. (2004) Oct4 is required for primordial germ cell survival. EMBO Reports, 5, 1078-1083.

61. Thomson, M., Liu, S.J., Zou, L.N., Smith, Z., Meissner, A., et al. (2011) Pluripotency factors in embryonic stem cells regulate differentiation into germ layers. Cell 145: 875-889.

62. Larsson NG, Wang J, Wilhelmsson H, Oldfors A, Rustin P, et al. (1998) Mitochondrial transcription factor $A$ is necessary for mtDNA maintenance and embryogenesis in mice. Nature Genetics, 18, 231-236.

63. Larsson, N.G., Oldfors, A., Holme, E., Clayton, D.A., (1994) Low levels of mitochondrial transcription factor A in mitochondrial DNA depletion. Biochemical and Biophysical Research Communications, 200, 1374-1381.

64. Poulton, J., Morten, K., Freeman-Emmerson, C., Potter, C., Sewry, C., et al. (1994) Deficiency of the human mitochondrial transcription factor h-mtTFA in infantile mitochondrial myopathy is associated with mtDNA depletion. Human Molecular Genetics, 3, 1763-1769.

65. Bhutani, N., Brady, J.J., Damian, M., Sacco, A., Corbel, S.Y., et al. (2010) Reprogramming towards pluripotency requires AID-dependent DNA demethylation. Nature, 463, 1042-1047.

66. Deng, W., (2010) AID in reprogramming: quick and efficient: identification of a key enzyme called AID, and its activity in DNA demethylation, may help to overcome a pivotal epigenetic barrier in reprogramming somatic cells toward pluripotency. Bioessays, 32, 385-387.

67. Cibelli, J.B., Campbell, K.H.S., Seidel, G.E., West, M.D., Lanza, R.P., (2002) The health profile of cloned animals. Nature Biotechnology, 20, 13-14.

68. Ying, Q.L., Wray, J., Nichols, J., Batlle-Morera, L., Doble, B., et al. (2008) The ground state of embryonic stem cell self-renewal. Nature, 453, 519-523.

69. Prigione, A., Fauler, B., Lurz, R., Lehrach, H., Adjaye, J., (2010) The senescence-related mitochondrial/oxidative stress pathway is repressed in human induced pluripotent stem cells. Stem Cells, 28, 721-733.

70. Armstrong, L., Tilgner, K., Saretzki, G., Atkinson, S.P., Stojkovic, M., et al. (2010) Human induced pluripotent stem cell lines show stress defense mechanisms and mitochondrial regulation similar to those of human embryonic stem cells. Stem Cells, 28, 661-673.

71. Birket MJ, Orr AL, Gerencser AA, Madden DT, Vitelli C, et al. (2011) A reduction in ATP demand and mitochondrial activity with neural differentiation of human embryonic stem cells. Journal of Cell Science, 124, 348-358. 
72. Tuppen, H.A., Hogan, V.E., He, L., Blakely, E.L., Worgan, L., et al. (2010) The p.M292T NDUFS2 mutation causes complex I-deficient Leigh syndrome in multiple families. Brain, 133, 2952-2963.

73. Munusamy, S., Saba, H., Mitchell, T., Megyesi, J.K., Brock, R.W., et al. (2009) Alteration of renal respiratory Complex-III during experimental type-1 diabetes. BMC Endocrine Disorders, 9, 2.

74. Rossignol, R., Faustin, B., Rocher, C., Malgat, M., Mazat, J.P., et al. (2003) Mitochondrial threshold effects. Biochemical Journal, 370, 751-762.

75. Jeppesen, T.D., Schwartz, M., Olsen, D.B., Vissing, J., (2003) Oxidative capacity correlates with muscle mutation load in mitochondrial myopathy. Annals of Neurology, 54, 86-92.

76. Trounce, I., (2000) Genetic control of oxidative phosphorylation and experimental models of defects. Human Reproduction, 15, 18-27.

77. Saladi, S.V., de la Sema, I.L., (2010) ATP dependent chromatin remodeling enzymes in embryonic stem cells. Stem Cell Rev 6: 62-73.

78. Wallace, D.C., Fan, W., (2010) Energetics, epigenetics, mitochondrial genetics. Mitochondrion, 10, $12-31$.

79. Dawid, I.B., (1974) 5-methylcytidylic acid: absence from mitochondrial DNA of frogs and HeLa cells. Science, 184, 80-81.

80. Groot, G.S., Kroon, A.M., (1979) Mitochondrial DNA from various organisms does not contain internally methylated cytosine in. Biochimica et Biophysica Acta, 564, 355-357.

81. Shock, L.S., Thakkar, P.V., Peterson, E.J., Moran, R.G., Taylor, S.M., (2011) DNA methyltransferase 1 , cytosine methylation, and cytosine hydroxymethylation in mammalian mitochondria. Proceedings of the National Academy of Science USA, 108, 3630-3635.

82. Oakes, C.C., La Salle, S., Smiraglia, D.J., Robaire, B., Trasler, J.M., (2007) Developmental acquisition of genome-wide DNA methylation occurs prior to meiosis in male germ cells. Developmental Biology, 307, 368-379. 


\section{Figure Legends}

Figure 1. The relationship between mtDNA copy number and pluripotency in reprogrammed cells. (A): Real- time PCR was performed to calculate the mtDNA copies/cell in undifferentiated iPS cells (lines 1-3), SC-ES cells (lines 1-4), ES-ES cells and their parental cell lines, MEFs (QS/Rosa26) and D3 ESCs (ESC); and NT-ES cells. All samples were normalised to $\beta$-actin. Bars represent means \pm s.e.m; significant differences between cell types are indicated $\left({ }^{* * *} \mathrm{P}<0.001\right)$. Expression of Oct4 $(\mathrm{B})$, Nanog (C) and Sox2 (D) was analysed by quantitative real-time PCR in mouse ESCs, iPS 2, SC-ES 1, ES-ES and NT-ES cells in their undifferentiated states and on Day 6 and 7 of differentiation. All samples were normalised to $\beta$-actin. Bars represent means \pm s.e.m; significant differences between cell types are indicated $\left({ }^{*} \mathrm{P}<0.05,{ }^{* *} \mathrm{P}<0.01,{ }^{* *} \mathrm{P}<0.001\right)$. (E) Altered pluripotent gene expression is correlated with elevated mtDNA copy number in reprogrammed cells. Pluripotent gene expression in ESCs, iPS, SC-ES and NT-ES was ranked (increased or decreased ranking based on relative expression against control ESCs) and plotted against mtDNA copy number. The correlation coefficient signifies the positive relationship between mtDNA and pluripotent gene expression in SC-ES and NTES. (F) Mitochondrial replication was examined by dual-labelling of BrdU (green) and MitoTracker (red) in undifferentiated (Und) ESCs, and SC-ES 1 and iPS 2 cells. ESCs and SC-ES cells demonstrated little or no replicating mtDNA while iPS cells contained large amounts of replicating mtDNA. Cell nuclei were visualised by DAPI (blue) staining. Scales bars $10 \mu \mathrm{m}$.

Figure 2. Expression of genes of differentiation in reprogrammed cells. ES and iPS 2, SC-ES 1, ES-ES and NT-ES cell differentiation was confirmed by RT-PCR analysis of germ layer markers: mesoderm (CD31 and VEGFR), ectoderm (Nestin and MAP2) and endoderm (AFP and FoxA2). $\beta$ actin was used to indicate equal loading.

Figure 3. MtDNA replication during in vitro differentiation. Embryoid bodies from (A) SC-ES 1 and (B) iPS 2 cells were analysed using real time PCR to determine the mtDNA copy number per cell during differentiation: Days 1 to 7 and 14 after the removal of LIF. All samples were normalised to $\beta$ actin and bars represent means \pm s.e.m. Significant differences between cell types are indicated $\left(^{*}\right.$ $\left.\mathrm{P}<0.05,{ }^{* *} \mathrm{P}<0.01,{ }^{* \star *} \mathrm{P}<0.001\right)$. (C) Dual-labelling with BrdU (24 hr; green) and MitoTracker (Red) on Day 1 of differentiation showed increased mtDNA replication and cytoplasmic expansion in iPS and 
SC-ES cells. Cell nuclei were visualised by DAPI (blue) staining. Scales bars $10 \mu \mathrm{m}$. Differentiating cells from (D) ES-ES (E) and NT-ES cells were analysed using real time as described in (A) on Days $1,6,7$ and 14 of in vitro differentiation. All samples were normalised to $\beta$-actin and bars represent means \pm s.e.m. Significant differences between cell types are indicated $\left({ }^{\star \star *} P<0.001\right)$.

Figure 4. The expression of the mtDNA-specific replication factors during in vitro differentiation of reprogrammed somatic cells. Expression of (A) Tfam, (B) Peo1, (C) mtSsbp1, (D) PolgA and (E) PolgB in ESC, SC-ES and iPS cells was analysed by quantitative real-time PCR on Days 1, 7 and 14 of differentiation. All samples were normalised to $\beta$-actin and values are expressed as a ratio of undifferentiated (Und) ESCs. Bars represent means \pm s.e.m. The significant differences between cell types and between days of differentiation within a particular cell line are indicated (* $\left.\mathrm{P}<0.05,{ }^{* *} \mathrm{P}<0.01,{ }^{* \star *} \mathrm{P}<0.001\right)$.

Figure 5. Treatment of pluripotent iPS cells with 5-Aza improves the regulation of mtDNA copy number during differentiation. Undifferentiated (und) (A) ESC cells and (B) iPS 2, (C) SC-ES 1, (D) ES-ES and (E) NT-ES cells were treated with $10 \mu \mathrm{M}$ 5-Aza for $72 \mathrm{hr}$ prior to differentiation and the effects on mtDNA copy were analysed by real time PCR on undifferentiated cells and Days 1, 6, 7 and 14 of in vitro differentiation. Significant differences between cell types are indicated $\left({ }^{*} \mathrm{P}<0.05 ;{ }^{*}\right.$ $\left.P<0.01 ;{ }^{* *} \mathrm{P}<0.001\right)$. ATP levels were analysed in ESC, iPS 2, SC-ES 1, ES-ES, and NT-ES pluripotent stem cells in normal ESC media or media supplemented with $10 \mu \mathrm{M}$ 5-Aza for $72 \mathrm{hr}(\mathrm{F})$. The values are relative luminescent units (RLU) normalised to ESCs from three independent experiments. Bars represent means \pm s.e.m; significant differences between treatments for each cell type are indicated $\left({ }^{*} \mathrm{P}<0.05\right)$. 

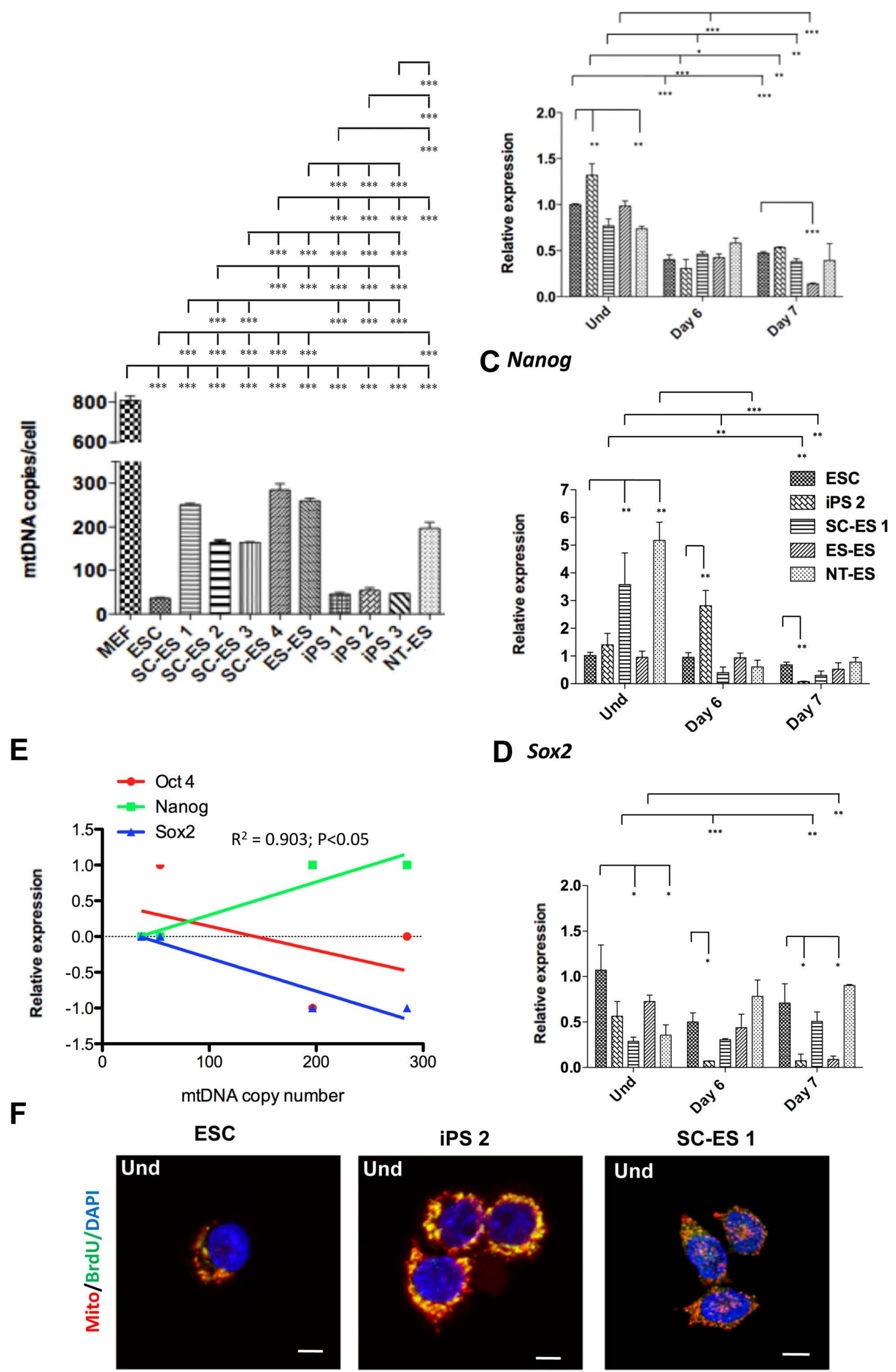


\section{Figure 2}

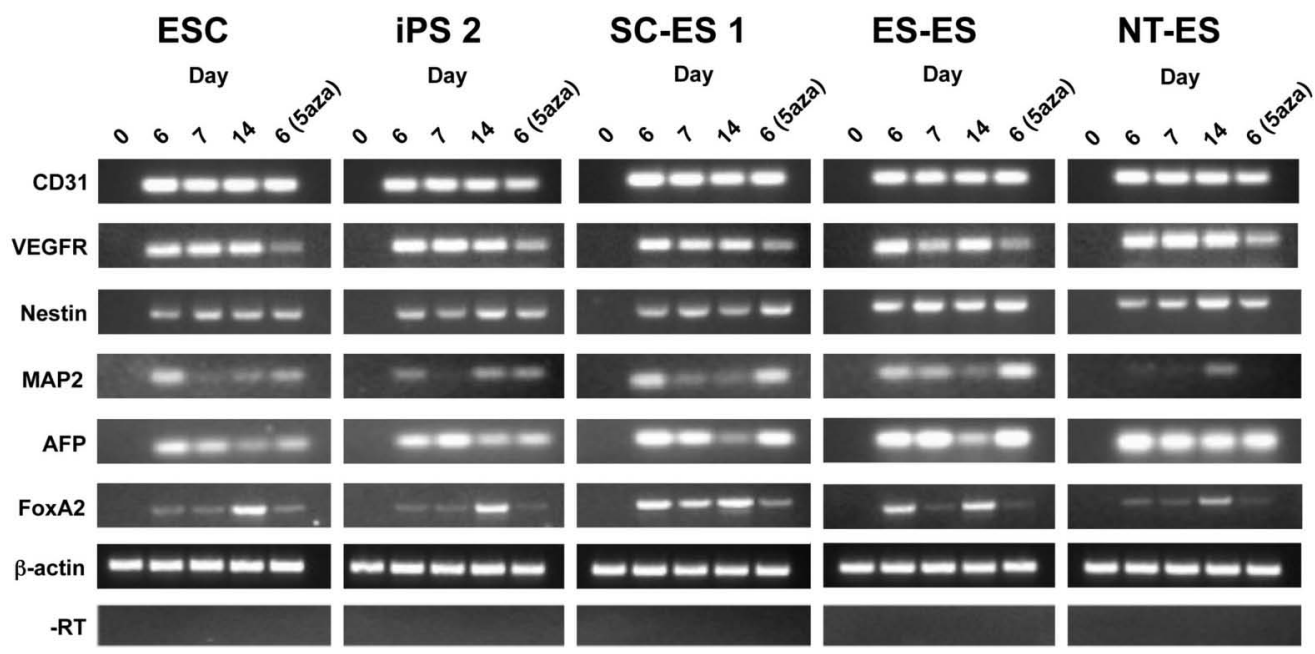




\section{Figure 4}

A tram
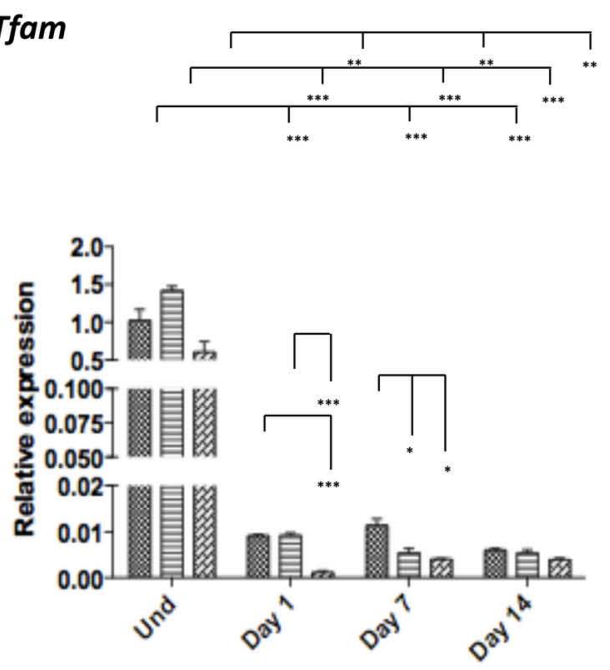

B Peo1

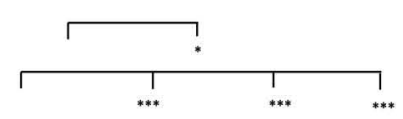

D PolgA

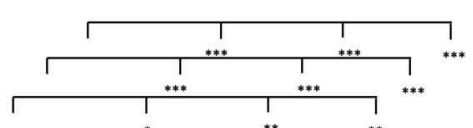

E PolgB

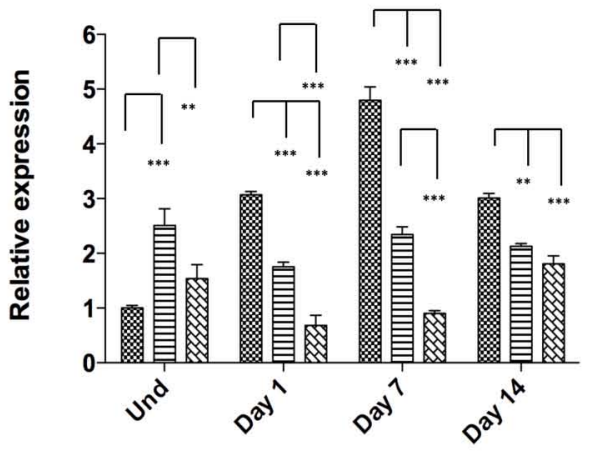

$C_{\text {mtSsbp1 }}$
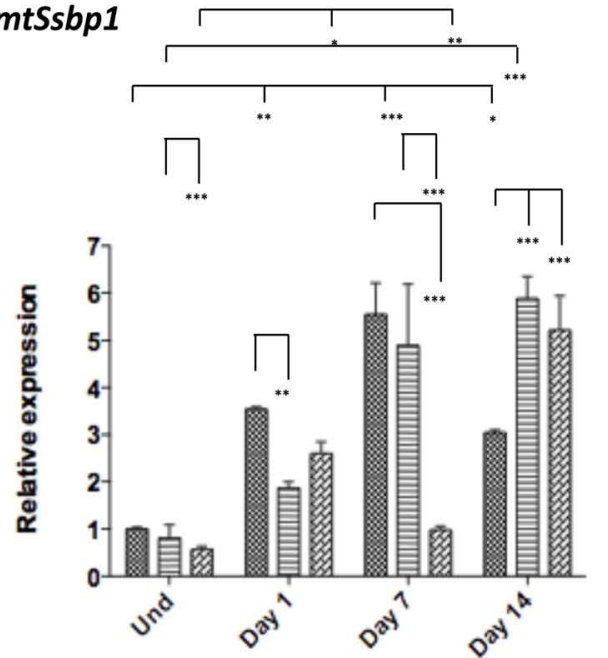
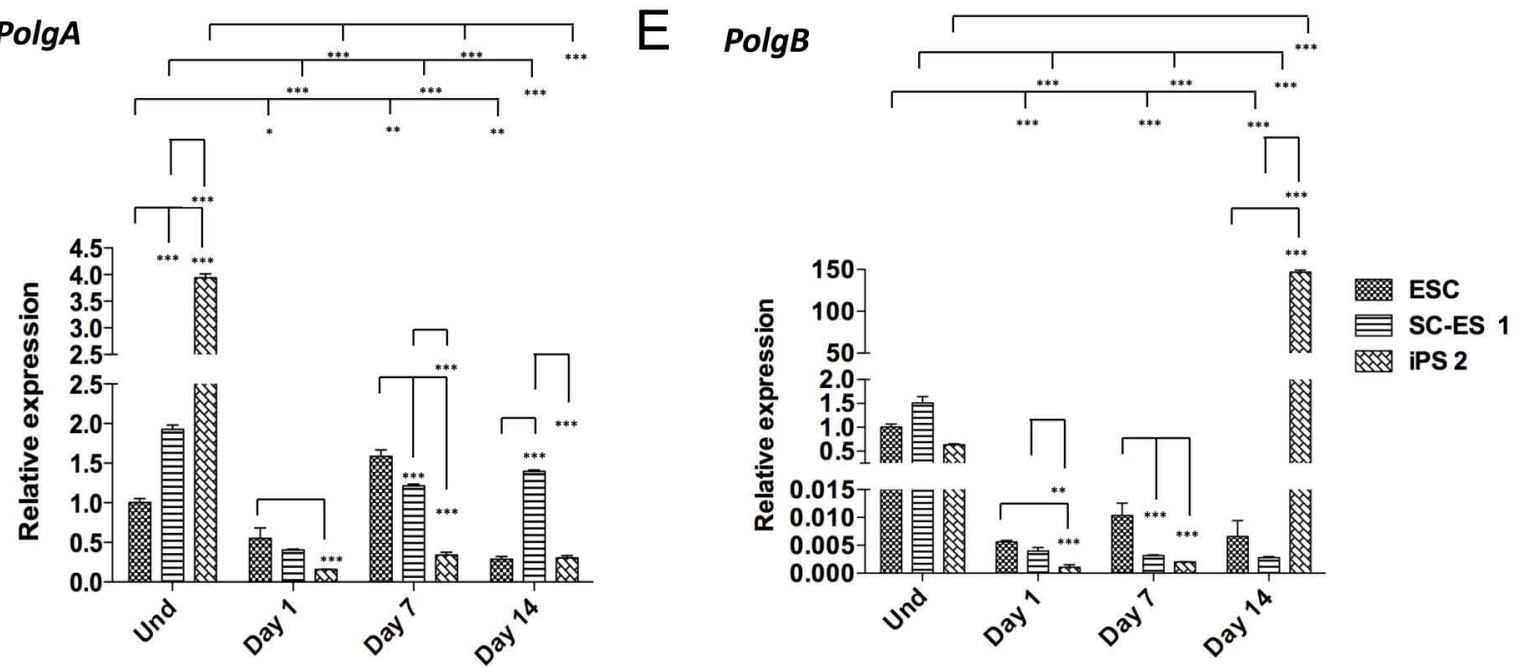


\section{Figure 5}

A MtDNA:

ESC (5Aza) differentiation

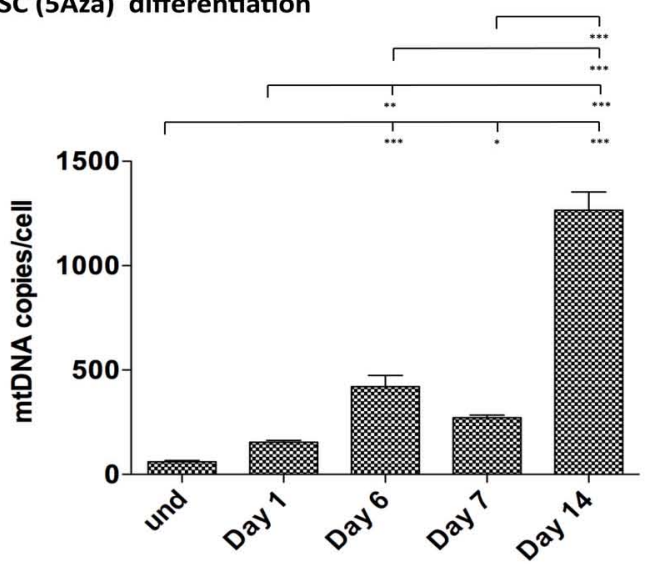

D MtDNA:

ES-ES (5aza) differentiation

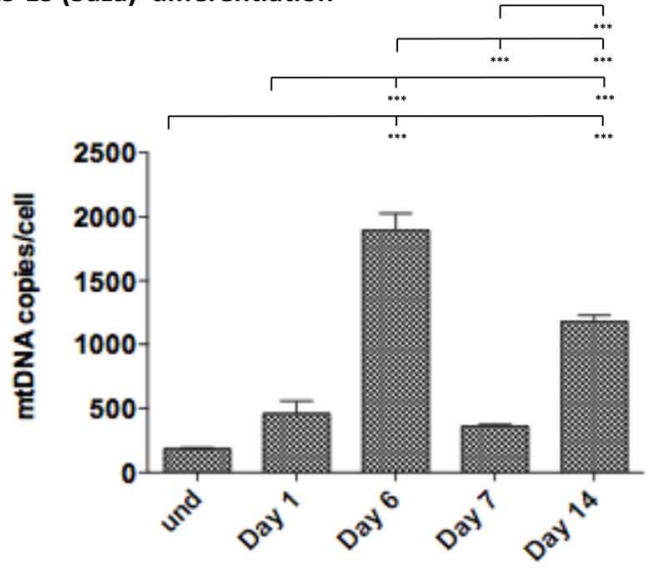

B MtDNA:

iPS 2 (5Aza) differentiation

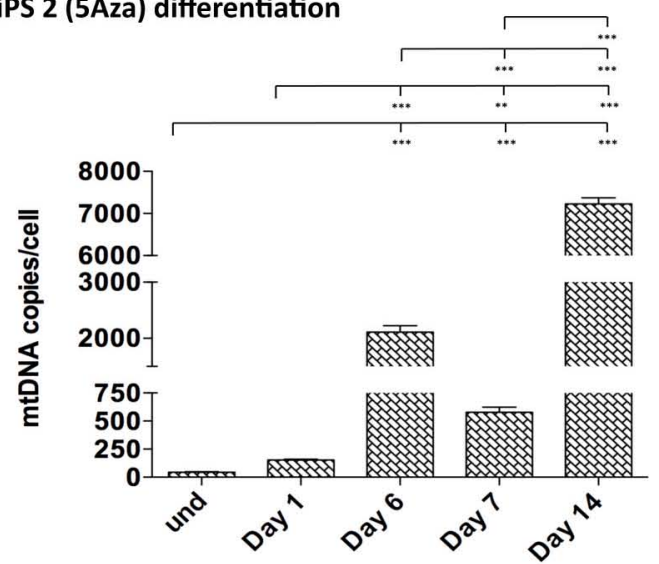

E MtDNA:

NT-ES (5aza) differentiation

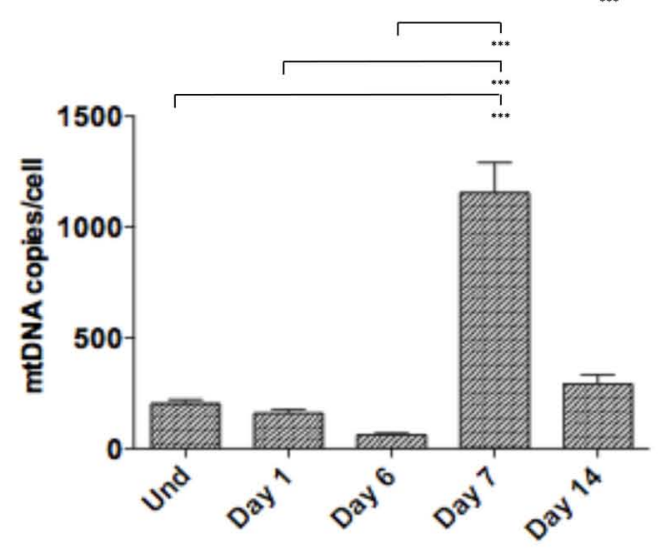

C MtDNA:

SC-ES 1 (5Aza) differentiation

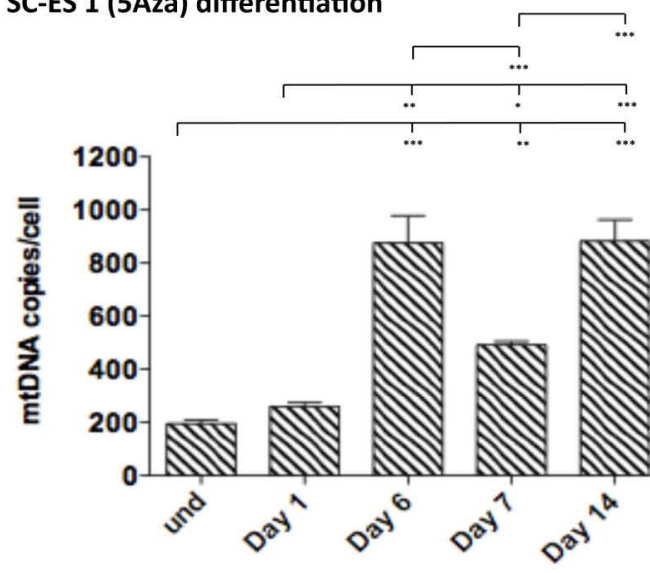

$\mathbf{F}$

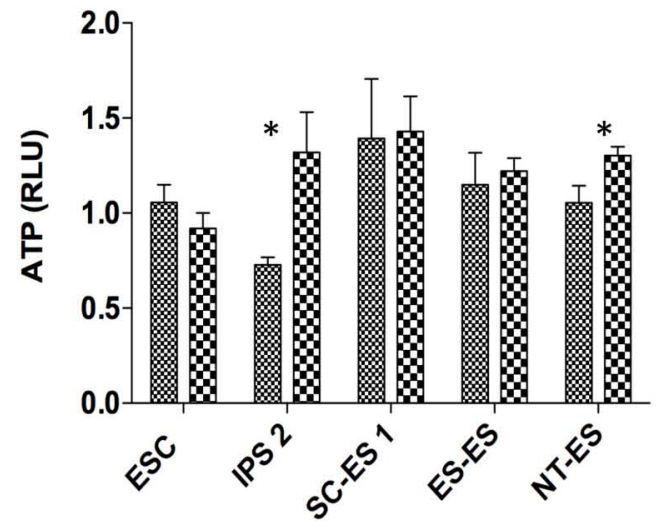

Untreated $\&$ 5-Aza 